\title{
Urban Land Expansion Simulation Considering the Diffusional and Aggregated Growth Simultaneously: A Case Study of Luoyang City
}

\author{
Renyang Wang ${ }^{1}$, Weishan $\mathrm{He}^{2}$, Dang $\mathrm{Wu}^{3}$, Lu Zhang ${ }^{4, *}$ and Yujia $\mathrm{Li}^{5, *}$ \\ 1 Research Institute of New Economic, Ningbo University of Finance \& Economics, Ningbo 315175, China; \\ wangrenyang@nbufe.edu.cn \\ 2 Anhui Transport Consulting \& Design Institute CO., LTD., Hefei 230088, China; hwshan333@163.com \\ 3 Wuhan Land Use and Urban Spatial Planning Research Center, Wuhan 430010, China; wudang@wlsp.org.cn \\ 4 School of Public Administration, Central China Normal University, Wuhan 430079, China \\ 5 College of Public Administration, Huazhong University of Science \& Technology, Wuhan 430074, China \\ * Correspondence: zhanglu54522@mail.ccnu.edu.cn (L.Z.); yujialee@hust.edu.cn (Y.L.)
}

Citation: Wang, R.; He, W.; Wu, D.; Zhang, L.; Li, Y. Urban Land

Expansion Simulation Considering the Diffusional and Aggregated Growth Simultaneously: A Case Study of Luoyang City. Sustainability 2021, 13, 9781. https://doi.org/ $10.3390 /$ su13179781

Academic Editor: Jacques Teller

Received: 12 July 2021

Accepted: 27 August 2021

Published: 31 August 2021

Publisher's Note: MDPI stays neutral with regard to jurisdictional claims in published maps and institutional affiliations.

Copyright: (c) 2021 by the authors. Licensee MDPI, Basel, Switzerland. This article is an open access article distributed under the terms and conditions of the Creative Commons Attribution (CC BY) license (https:// creativecommons.org/licenses/by/ $4.0 /)$.

\begin{abstract}
Restricted by urban development stages, natural conditions, urban form and structure, diffusional growth occupies a large proportion of area in many cities. Traditional cellular automata (CA) has been widely applied in urban growth studies because it can simulate complex system evolution with simple rules. However, due to the limitation of neighborhood conditions, it is insufficient for simulating urban diffusional growth process. A maximum entropy mode was used to estimate three layers of probability spaces: the probability layer of cell transformation from non-urban status to urban status (PLCT), the probability layer for aggregated growth (PLAP), and the probability layer for diffusional growth (PLOP). At the same time, a maxent category selected CA model (MaxEnt-CSCA) was designed to simulate aggregated and diffusional urban expansion processes simultaneously. Luoyang City, with a large proportion of diffusional urban expansion (65.29\% in 2009-2018), was used to test the effectiveness of MaxEnt-CSCA. The results showed that: (1) MaxEnt-CSCA accurately simulated aggregated growth of $47.40 \%$ and diffusional growth of $37.13 \%$ in Luoyang from 2009 to 2018, and the overall Kappa coefficient was 0.78; (2) The prediction results for 2035 showed that future urban expansion will mainly take place in Luolong District and the counties around the main urban area, and the distribution pattern of Luolong District will change from the relative diffusion state to the aggregation stage. This paper also discusses the applicable areas of MaxEnt-CSCA and illustrates the importance of selecting an appropriate urban expansion model in a region with a large amount of diffusional growth.
\end{abstract}

Keywords: diffusional urban expansion; maximum information entropy; cellular automata; Luoyang City

\section{Introduction}

Cellular automata (CA) has been widely used in the field of urban expansion modeling because it can simulate complex macro-scale evolutions using simple micro-scale conversion rules [1-3]. Limited by the rules of neighboring cells, most existing urban expansion CA models only consider the situation where the urban land is added around existing urban land $[4,5]$, and can actually simulate an urban plane extending in a specific direction. However, in the real world, urban built-up space is often developed piece by piece with a diffusion form and may not be alongside the original urban land patch [6]. As a result, simply describing the dynamic changes of urban expansion as a consecutive extended surface is very insufficient. Urban land dynamics are actually a collection of complex evolution forms containing internally connected dots, the expansion of planes and the integrated interactions between dots and planes [7,8]. The growth of "dots" occurs in different periods of urban development [6]. In the early stage of urban expansion, a large 
number of small construction patterns emerge quickly around the original main city pattern $[9,10]$, which manifest as "diffusion" and can be abstracted as "dots" in growth $[5,11]$. At the stable development stage, large urban blocks, newly constructed satellite cities, development zones, and small towns commonly appear, which are significantly smaller and are not spatially connected to the large urban blocks [12]. This phenomenon of disjoint and spatial separation can also be abstractly referred to as "dot" growth. In the traditional CA model, none of these "dot" growth processes can be effectively simulated $[5,6]$.

According to the landscape expansion index [13-15], newly expanded urban patches can be classified into either edge, infill or outlying types [10,16]. Edge expansion refers to the extension of urban built-up areas along the original urban patch and infill expansion occurs in the internal voids patches, both of which can make the original urban built-up area more compact. Outlying expansion is a leapfrog development of land far away from the original urban land and can make the city more diffusional [7]. Few scholars have tried to improve traditional CA models to simulate outlying-type expansion. Clarke proposed an improved CA model called SLEUTH, which can randomly select newly generated points to generate extensional expansion [11], but the proliferation of new urban patches is not completely random. In fact, the occurrence of the new patches is the result of multiple driving forces [5]. As a result, SLEUTH may not be able to simulate a real urban proliferation scenario. Liu et al. proposed the SMDUGT model to simulate diffusion and aggregation in urban morphology evolution [6]. The basic idea of SMDUGT is to divide the expansion candidate areas of different expansion types based on regression results, and then consider neighborhood factors to construct the adjacent (edge and infill) and outlying (outlying) types. The cell conversion rules in the candidate regions for land-type expansion are integrated into the cellular automata model to obtain simulation methods for different types of regions. However, SMDUGT cannot determine where the "seed points" of diffusion growth are.

The maximum entropy model (MaxEnt) is a niche model based on the maximum entropy principle [17]. It takes research areas as all possible-probability distribution and finds the most likely distribution under the maximum entropy principle according to constraints given by environmental variables [18-21]. It finds an optimal distribution using single-period sample information to select the distribution with the largest entropy from various environmental variables and uses it to predict the probability of research object appearance [22]. Thanks to its simplicity and practicality, the model's application range has rapidly expanded from biological research to other fields. In the field of urban expansion simulation, Zhang et al., coupled the maximum entropy model with the cellular automata (CA), and proposed a new urban expansion model called Maxent-CA to analyze single-period training samples and obtain urban land growth suitability [18]. The proposed Maxent-CA model overcame the traditional CA model requirement of parameter correction from multi-phase remote sensing images and the request for partitioning when largearea simulations are needed. However, the simultaneous simulation of diffusional and aggregated growth processes could not be achieved in their study.

This paper attempted to use MaxEnt model to estimate the three-layers probability space: the probability layer of cell transformation from non-urban to urban status (PLCT), the probability layer of suitability distribution for adjacent patches (PLAP), and the probability layer of suitability distribution for outlying patches (PLOP). Of these, PLCT is used to sort the priority order of cells to be selected, and PLAP and PLOP are used to determine the type of the selected cell. Based on the three-layer probability space, the Maximum Entropy Category Selected CA model (Maxent Category Selected CA model, MaxEnt-CSCA) was proposed. The great advantage of the model is that it can simulate diffusional and aggregated growth simultaneously, which is closer to a real-world situation and greatly improves the model's predictive accuracy. The MaxEnt-CSCA model adopts a "divide and conquer" design paradigm, which distinguishes different features of the adjacent and outlying urban expansion type and integrates their information. First, the model identifies urban patch expansion types using the landscape expansion index, and 
then the MaxEnt model generates the three-layers probability space (PLCT, PLAP and PLOP), depending on patch geographic location and various kinds of social and natural environmental variables. Next, according to the different growth characteristics, two sets of local cell evolution rules are designed and integrated to the model. Finally, in the CA iterative simulation stage, the category significance (adjacent or outlying) of each geographic cell is determined according to the probability of the suitability layer and the corresponding local cell evolution rule is selected to determine the conversion state. The innovation of the MaxEnt-CSCA model is to improve the deficiencies of traditional CA in simulating outlying urban expansion, that is, to perform ternary instead of binary land use simulation (non-urban, urban-adjacent, and urban-outlying) which allows simultaneous simulation of diffusional and aggregated growth.

Luoyang City, located in Henan Province, China, was selected to test the effectiveness of MaxEnt-CSCA model. From 2009-2018, Luoyang city expanded significantly, and the growth of outlying urban patches was very obvious. According to the Chinese land use survey data, the percentage of outlying growth was $65.29 \%$. Therefore, traditional CA models were unable to accurately capture the urban growth trajectory of Luoyang City. The objective of this paper is to verify the practicability and effectiveness of maxent-CSCA in modeling urban dynamic evolution processes where diffusional growth accounts for a significant proportion, and call on scholars related to urban modeling research to pay more attention to different types of urban expansion in the process of urban development. Our expected result is that Maxent-CSCA can achieve higher simulation accuracy than Maxent-CA and Base-CA without considering diffusional process, and thus is more suitable for the prediction and simulation of the future urban landscape of Luoyang city in 2035.

\section{Study Area and Data}

Luoyang city is located in the west of Henan Province, bordering Zhengzhou, the capital of Henan Province. Its latitude ranges from $118^{\circ} 08^{\prime} \mathrm{E}$ to $112^{\circ} 59^{\prime} \mathrm{E}$ and the longitude range ranges from $33^{\circ} 39^{\prime} \mathrm{N}$ to $35^{\circ} 05^{\prime} \mathrm{N}$. The total population of the city is 7.1702 million, of which 4.091 million live in urban areas and the urbanization level is $59.10 \%$. The population density is 455 people $/ \mathrm{km}^{2}$ and GDP is 503.49 billion yuan by 2019. Luoyang City has 15 county-level administrative districts in total. However, considering that urban built-up areas are mostly located in the main city, six administrative districts in the main urban area were selected as the research focus. The distribution of built-up areas in 2009 and 2018 is shown in Figure 1. Table 1 shows the increase in built-up area over the years from 2009 to 2018 in the study area. Using linear regression, the built-up area was predicted to be about $266.39 \mathrm{~km}^{2}$ in 2035 . In 2009 , the built-up area in the main city accounted for $17.49 \%$ of the total administrative area. By 2018 , this was $21.78 \%$, and by 2035 it could reach $30.28 \%$. The built-up area is expected to continue to expand, and the rate of urban expansion will gradually increase over time.

The 2009-2018 built-up area data was from the Chinese second national land survey data. Built-up area data for 2010-2018 was derived from a year-by-year land change survey database based on second national land survey data in 2009. These time series data had the same survey standard, with a coordinate system of CGCS_2000 and in vector format. Data surveys were led by national institutions to accurately grasp land use types and changes. Due to the combination of remote sensing images, aerial photography, and a large number of field surveys, this data source has higher authority and accuracy than the traditional land cover data based only on remote sensing image interpretation. The basic geographic data involved in this article, such as road networks, government centers, airports, water bodies, and commercial distribution, were directly extracted from this database (see these elements in Figure 1). The population density data was derived from the global $1 \mathrm{~km}$ grid distribution database by the National Oak Ridge Laboratory [12]. The $1 \mathrm{~km}$ resolution overcomes statistical deficiencies based on administrative district surveys and can express population distribution characteristics on a more refined scale [23,24]. The land price data was from the national land transaction database. The information contained in the database 
for each transferred land piece included the location, the specified use, the transfer area and the transfer price. The location Geocode interface provided by Gaode Maps was used to obtain the spatial positions of 1818 parcels sold in the study area from 2009 to 2018, and the average price was calculated according to sold area and price. The final spatially continuous average price distribution of land sales from 2009 to 2018 was generated using the spatial interpolation tool in ArcGIS.

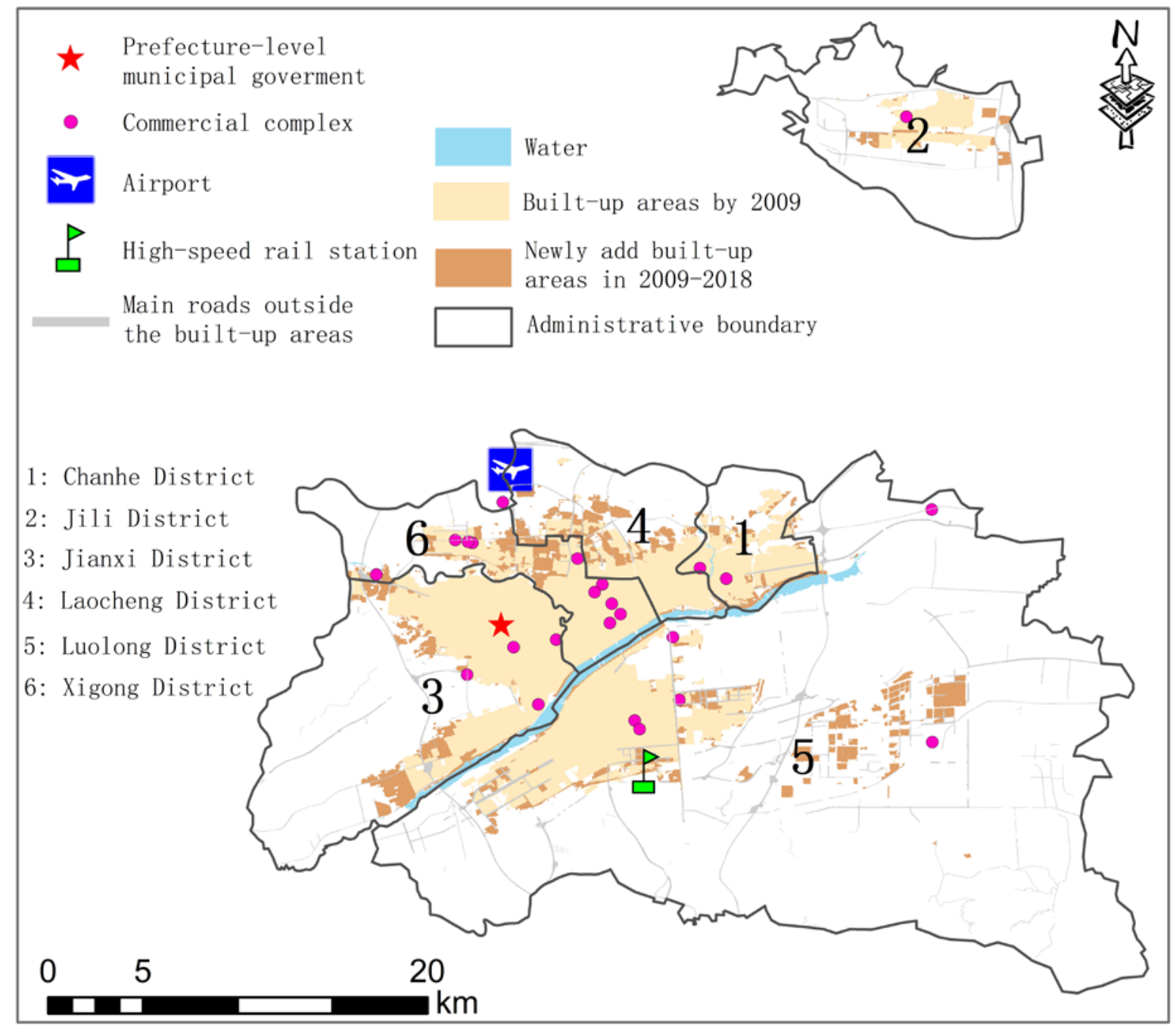

Figure 1. Distribution of built-up and basic geographical elements in the study area.

Table 1. Built-up area in the main city of Luoyang from 2009 to 2018 and projected area in 2035.

\begin{tabular}{ccccccccccccc}
\hline Year & 2009 & 2010 & 2011 & 2012 & 2013 & 2014 & 2015 & 2016 & 2017 & 2018 & $\begin{array}{c}\text { 2035 } \\
\text { (Predicted) }\end{array}$ \\
\hline Area $\left(\mathrm{km}^{2}\right)$ & 153.86 & 161.79 & 166.60 & 173.90 & 178.82 & 180.61 & 181.57 & 189.93 & 190.99 & 191.59 & 266.39 \\
\hline
\end{tabular}

\section{Models and Methods}

The MaxEnt-CSCA model consists of two parts: model training and spatial simulation. In the model training stage, a landscape expansion index was used to identify the expansion type of new built-up patches and several environmental variables based on spatial proximity, socio-economic development, and topography. These were selected as the driving factors that affected expansion type. Then, urban patch sample data of different types was inputted to the MaxEnt model and the three-layers probability space (PLCT, PLAP, 
and PLOP) was obtained by training these data to find the maximum entropy distribution. In the model training stage, all cell priorities were sorted according to their corresponding values in PLCT space. Higher values indicated higher cell selection priorities. For a selected cell, their corresponding value in PLAP and PLOP space determined whether it was an outlying type or adjacent type. The neighborhood function (NF), random factors (RF) and variability of different land types (VDT) were considered to create conversion rules for different urban patch types The details about NF and RF have been explained in detail in literature [18], and VDT will be further explained in Section 3.3. Finally, the model's parameters were corrected and used to simulate the dynamic process of future urban expansion. The MaxEnt-CSCA model framework is shown in Figure 2.

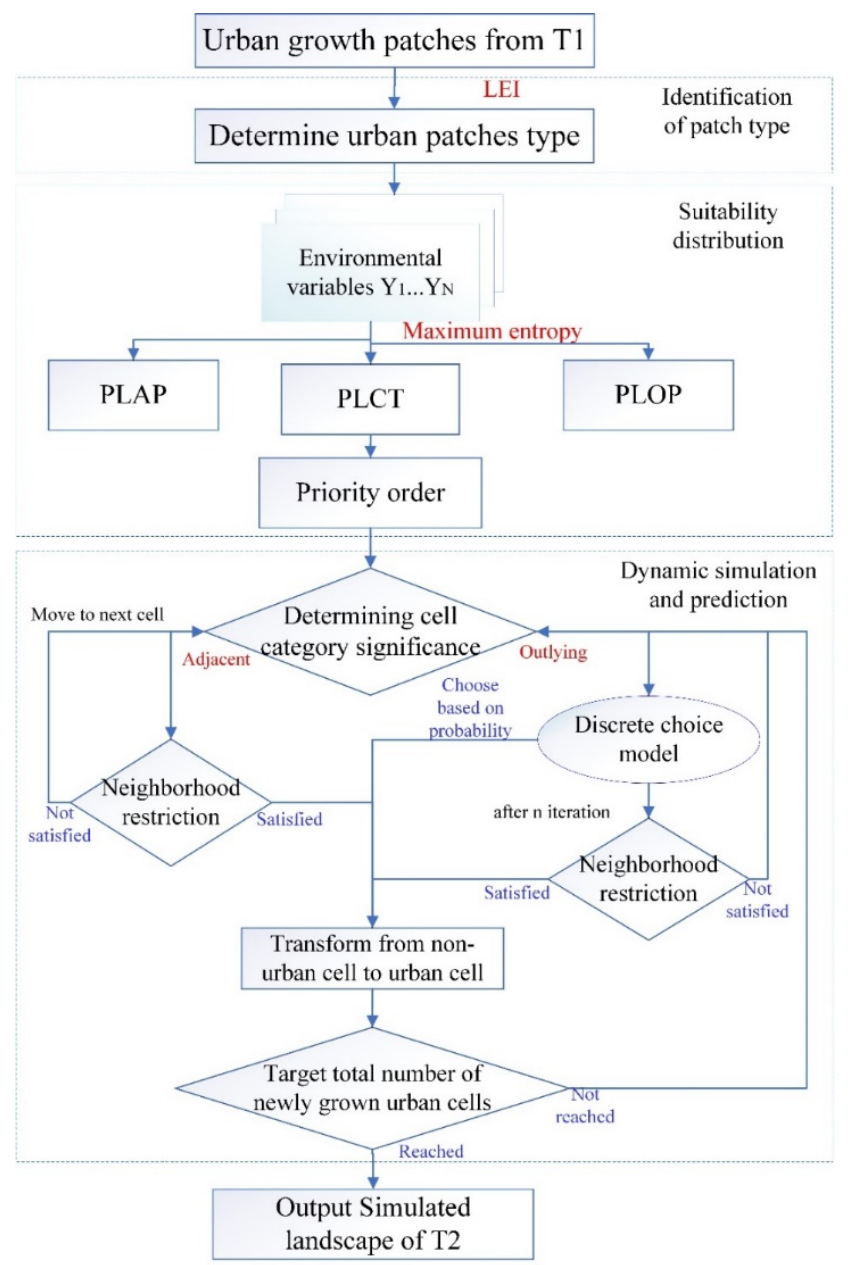

Figure 2. Algorithm flow of MaxEnt-CSCA model.

\subsection{Identification of Patch Type}

Urban growth type (UGT) in this article included two categories: adjacent and outlying. Adjacent UGTs refer to the expansion that transforms rural land villages within a city or cultivates unused land around the city into urban built-up area. In this type of expansion, new urban area is spatially adjacent to the original city. Outlying UGTs refer to when new urban area is spatially disconnected from the original built-up area, but the two areas are still functionally interconnected through transportation. Considering the definition of the relationship between the new and original urban patches in the literature [10], a landscape expansion index (LEI) was used to determine the type of each newly added urban patch and it was calculated as follows:

$$
L E I=\frac{A_{o}}{A_{o}+A_{v}} \times 100
$$


In which, $A_{o}$ is the intersecting area of a new urban patch with the original patch. $A_{v}$ is the intersecting area of a new patch and non-urban land. According to the definition of $L E I$, when $L E I=0$, the new urban patch is an outlying expansion. When $L E I>0$, it is adjacent expansion.

\subsection{MaxEnt Principle and Environmental Variables Seletion}

The principle of maximum entropy (MaxEnt) is used to obtain a probability distribution with the highest entropy, which means the distribution is the most scattered or the most uniform, after satisfying the known partial information. The principle and criteria were first proposed in information theory [18], and soon were introduced and applied to various research fields. Typically, the total size of the target predicted object is unknown, so the absolute occurrence rate on a specific geographical cell is meaningless and the relative occurrence rate was introduced. In our settings, the relative occurrence rate $P\left(z\left(x_{i}\right)\right)$ given by Maxent represents the probability that cell $\mathrm{i}$ is more likely to be transformed into urban (or urban-adjacent or urban-outlying) than other cells over the study area, which is calculated as follows:

$$
P\left(z\left(x_{i}\right)\right)=\frac{\exp \left(z\left(x_{i}\right) \lambda\right)}{\sum_{i} \exp \left(z\left(x_{i}\right) \lambda\right)}
$$

where $z\left(x_{i}\right) \lambda=z_{1}\left(x_{i}\right) \lambda_{1}+z_{2}\left(x_{i}\right) \lambda_{2}+\ldots+z_{K}\left(x_{i}\right) \lambda_{K}$, where $x_{i}$ is the position of cell $\mathrm{i}$, $z\left(x_{i}\right)$ is the vector of environmental variables at location $x_{i}$ of cell $i$ and $\lambda$ is a vector of regression coefficients learned from the data, which indicates the relative importance of the different environmental variables. The sum of the relative occurrence rate over all cells in the study area is 1 , i.e., $\sum_{i=1}^{N} P\left(z\left(x_{i}\right)\right)=1$, because the denominator normalizes the relative occurrence rates.

According to the definition, the relative entropy of the target object's presence is calculated as:

$$
H(x)=\sum_{i=1}^{N} P\left(z\left(x_{i}\right)\right) \log \frac{P\left(z\left(x_{i}\right)\right)}{Q\left(x_{i}\right)}
$$

where $Q(x i)$ is the prior distribution, which reflects user expectations about the distribution before accounting for the known data. Here all the grid cells are a priori considered equally likely to be at the presence of the target object and $Q(x i)$ was set to uniform distribution to maximize entropy in geographical space. However, in order to ensure the consistency between the predicted distribution and the known data, constraints were made so that the predicted distribution corresponded to the known known urban (or urban-adjacent or urban-outlying) cell distribution, in terms of environmental variables' moments such as mean and variance. Since the exact position $\dot{x}_{1}, \dot{x}_{2}, \ldots, \dot{x}_{m}$ of all urban cells present in a given year is known and MaxEnt assumes that the existence probability of the target object at a certain location (cell $\mathrm{i}$ ) is a function of $\mathrm{K}$ environmental predictors, the constraints formula can be written as follows for each environmental predictor $z_{k}$ :

$$
\sum_{i=1}^{N} z_{k}\left(x_{i}\right) P\left(z\left(x_{i}\right)\right)=\frac{1}{M} \sum_{m=1}^{M} z_{k}\left(\dot{x}_{m}\right)
$$

where $z\left(x_{i}\right)$ is the vector of environmental variables at location $x_{i}$ of cell i mentioned above, $\dot{x}_{m}$ is the position of the known urban cells present in a given year, and $\frac{1}{M} \sum_{m=1}^{M} z_{k}\left(\dot{x}_{m}\right)$ gives the mean of the $k^{\text {th }}$ environmental variable of the known urban cells. The mean of the environmental value of the predicted distribution on the left side of Equation (4) is constrained to be the same value of the mean of the environmental variable at the observed locations on the right side. After the necessary constraints were generated, the maxent software minimized relative entropy within environmental space. By continuously adjusting the parameter values through a random seed generation algorithm, the optimal solution was found and output from the software. Because samples were only trained once and could introduce accidental errors, this study used the average of multiple training outcomes as the final results (PLCT, PLAP, and PLOP). 
Regarding the selection of environmental variables, we referred to the previous literature and local characteristics of the study area to select spatial proximity (distance to political centers, major roads, airports, existing built-up areas, commercial centers, and high-speed rail stations), socioeconomic factors, including two variables, population density and newly added enterprise density for 2009-2018 [5,25-28]. The selection of spatial proximity has been explained in detail in previous studies and is not repeated. Although population density may not directly increase UGT, it can indirectly affect the formation of UGT through the cost of land development, which proved to be a very important factor [29]. For example, population density is related to the labor cost of demolition and resettlement. The higher the population density, the higher the investment that is compensated to the relocated population. For other density variables, such as areas with high road density with relatively complete infrastructure, additional investment required for land development will be significantly reduced, which proved beneficial to outlying growth [29]. The statistics of the above eight indicators are shown in Table 2.

Table 2. Statistics of eight environment variables.

\begin{tabular}{ccccc}
\hline Variable & Description & Minimum & Maximum & Standard Deviation \\
\hline toBuilt & Distance to original built-up areas $(\mathrm{km})$ & 0 & 18.29 & 4.73 \\
toRoad & Distance to main road $(\mathrm{km})$ & 0 & 26.28 & 1.60 \\
toPCenter & Distance to political center $(\mathrm{km})$ & 2.12 & 34.17 & 4.92 \\
toCCenter & Distance to commercial center $(\mathrm{km})$ & 2.13 & 38.14 & 6.82 \\
toAirport & Distance to airport $(\mathrm{km})$ & 0 & 41.33 & 7.60 \\
toHRailStation & Distance to high-speed rail station $(\mathrm{km})$ & 0 & 4.31 \\
GDPDensity & GDP density (10,000 yuan $\left./ \mathrm{km}^{2}\right)$ & 259.48 & 1059.81 & 2061.79 \\
NFDensity & Newly added firm in 2009-2018 in $1 \mathrm{~km}^{2}$ & 0 & 35.89 \\
\hline
\end{tabular}

\subsection{Dynamic Simulation and Prediction}

After the probability space and target number were calculated, the MaxEnt-CSCA model was ready to be simulated. We sorted the suitability probability of each potential cell from large to small, and selected cells with high suitability probability in each round of iteration. This iteration method is called SortCA in the literature [5], which can ensure that cells with higher suitability potential have a higher conversion probability (from non-urban to urban). For each selected cell, if it is the cell to be expanded in the study area (assuming the number of rows and columns were $x, y$, respectively), the significance of its expansion type will be checked. If the numerical value of the suitable probability of the adjacent category was greater than or equal to the outlying category, i.e., PLAP $[x, y] \geq \operatorname{PLOP}[x, y]$, then the cell was judged to be the adjacent type significant, otherwise, the cell was judged to be the outlying category significant.

For a cell significant in the adjacent category, it was greatly affected by neighboring cell land use. If there were more surrounding built-up cells, it was easier for a cell to be transformed into a built-up cell. Here we defined the local cell rule. If it met the requirements that the minimum number of urban cells in the Moore neighborhood exceeded a threshold, the state of the cell was transformed from a non-urban cell to an urban cell at the probability of PLAP $[x, y]$. If not, the central cell moved to the next selected cell according to priority order.

For a cell significant in the outlying category, in the first $\mathrm{n}$ iterations ( $\mathrm{n}$ was the set threshold), the expansion of the city was simulated based on the discrete choice model, which allowed cell transfers based on probability without meeting the minimum number of urban cells in the Moore neighborhood. According to literature [30], the probability of position i being selected was equal to the probability that position i utility was greater than or equal to the utility of any other alternative position $\mathrm{j}$, namely:

$$
P(i)=P(U(i) \geq U(j))=\frac{\exp (U(i))}{\sum_{i=1}^{n} \exp (U(i))}
$$


In the formula: $U$ represents the utility function. In this study it represented the outlying suitability probability function, and $\sum_{i=1}^{n} \exp (U(i))$ was the sum of the candidate location utility exponential functions. The discrete selection model showed that in a location with higher probability of outlying suitability, it was more likely that a cell would be randomly selected and developed. The growth seeds of outlying category UGTs were generated according to PLOP $[x, y]$ probabilities. After generating random seeds in the first $\mathrm{n}$ iterations, the condition of the minimum number of city cells in the neighborhood was added to restrict the growth of outlying-significant cells and prevent disorderly, sporadic built-up growth.

The condition to terminate the simulation was to determine whether the number of newly added urban cells reached the target urban cell quantity. If the condition was met, the simulation process ended and output a layout plan for new urban land. If the condition was not met, the next iteration began from the first cell in priority order again until the quantity of newly added construction cells was reached. The Kappa coefficient is a measure of the difference between the model's prediction result and a random guess result, and values range between 0 and 1 . The higher the kappa coefficient, the higher the accuracy of the model.

\section{Results}

\subsection{Statistical Analysis of UGT}

Restricted by different natural, social and economic factors in the specific geographical location, the urban expansion size and type differed from region to region in the main urban area of Luoyang. By calculating LEI, UGTs from 2009 to 2018 in Luoyang were classified and the statistics of newly-added patches in each administrative district of the main urban area were obtained (see Figure 3). According to the UGT identification, 2373 new urban patches were found from 2009 to 2018, of which 1463 were outlying UGT patches with an area of about $24.64 \mathrm{~km}^{2}$, accounting for $65.29 \%$ of the new urban area. Adjacent UGTs increased by 2293 patches with an area of about $13.10 \mathrm{~km}^{2}$, accounting for $34.71 \%$ of the new urban area. Urban area growth (quantity statistics and spatial distribution) in the six districts of the main urban area of Luoyang City is shown in Table 3 and Figure 3.

Table 3. Urban area growth in the six districts of the main urban area of Luoyang City.

\begin{tabular}{ccccccc}
\hline Type & $\begin{array}{c}\text { Chanhe District } \\
\mathbf{( 1 )}\end{array}$ & $\begin{array}{c}\text { Jili District } \\
\mathbf{( 2 )}\end{array}$ & $\begin{array}{c}\text { Jianxi District } \\
\mathbf{( 3 )}\end{array}$ & $\begin{array}{c}\text { Laocheng } \\
\text { District } \\
\mathbf{( 4 )}\end{array}$ & $\begin{array}{c}\text { Luolong } \\
\text { District } \\
\mathbf{( 5 )}\end{array}$ & $\begin{array}{c}\text { Xigong District } \\
\text { (6) }\end{array}$ \\
\hline Outlying & 0.046 & 1.906 & 2.684 & 1.600 & 17.394 & 1.007 \\
Adjacent & 0.783 & 1.310 & 2.948 & 2.318 & 4.200 & 1.531 \\
\hline
\end{tabular}

As can be seen in Table 3 and Figure 3, Luolong District (5) had the largest increase in built-up area $\left(21.594 \mathrm{~km}^{2}\right)$, much more than other regions, followed by Jianxi District (3), Laocheng District (4) and Jili District (2). The newly built-up areas of these top four administrative units were all larger than $3 \mathrm{~km}^{2}$ while Chanhe District (1) had the smallest newly built-up area, less than $1 \mathrm{~km}^{2}$. It is worth noting that the adjacent UGT areas in four districts, namely Chanhe, Jianxi, Laocheng and Xigong, were larger than the outlying UGT areas. This was because these four districts were geographically adjacent and had relatively small jurisdictions, in which the proportion of built-up land was already high. Due to space constraints, it was difficult for the cities in these four regions to develop built-up areas in a diffusion manner. Luolong District (5), as the largest administrative district in the main urban area, had the space to build new satellite cities and industrial parks away from the existing built-up areas. In fact, Luolong District's outlying UGTs accounted for more than $80.55 \%$ in $2009-2018$ and the characteristics of diffusion growth in this district were very obvious. 


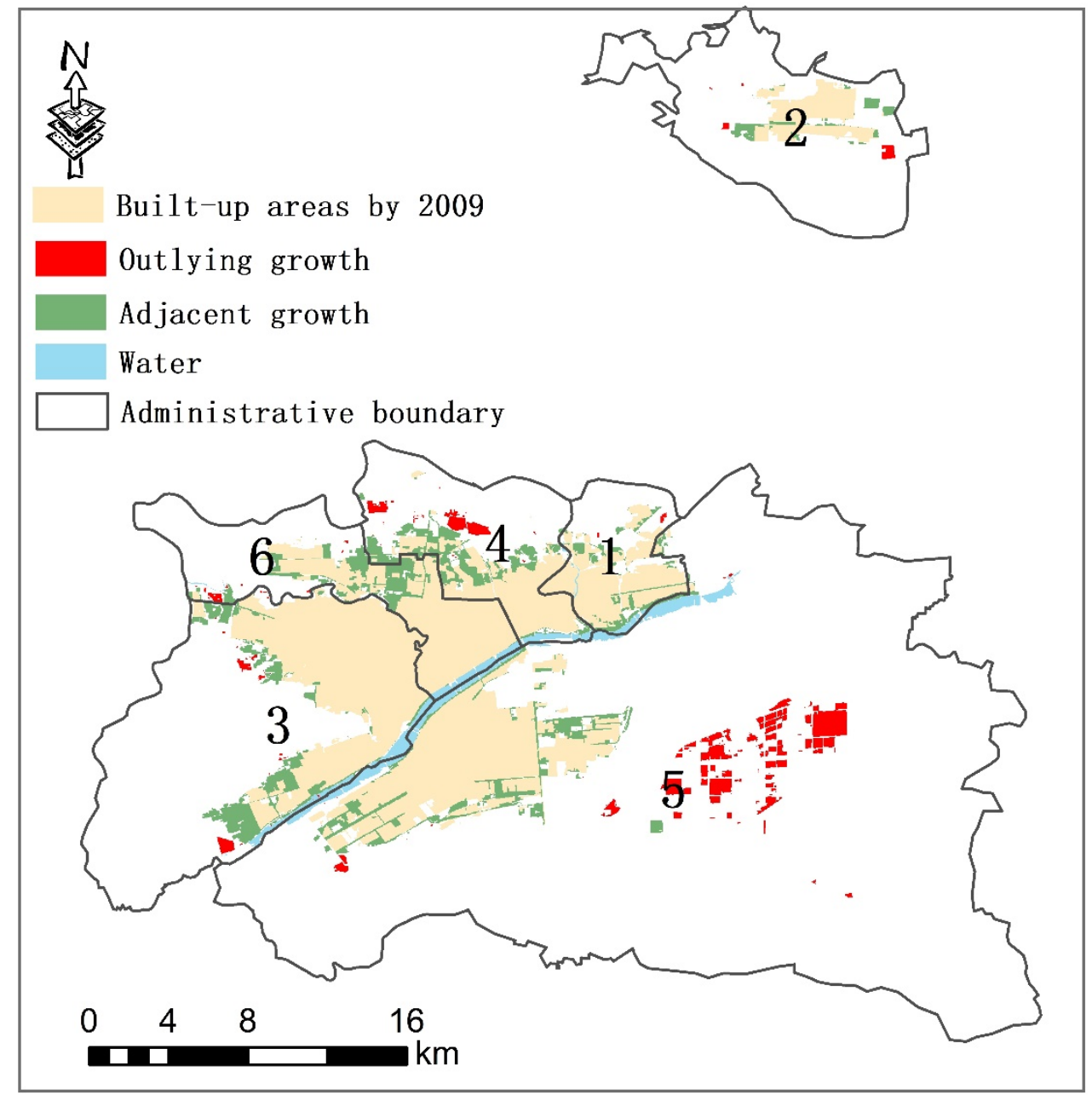

Figure 3. Spatial distribution of UGTs for new urban patches in the six districts of the main urban area of Luoyang City.

\subsection{Suitability of UGT and Variable Contributions}

Figure 4 shows the distributions of PLAP and PLOP based on the MaxEnt model. As can be seen in the PLOP figure, high values were distributed in areas away from the original built-up areas. Statistically, 77.37\% cells with PLOP were greater than 0.70 and were located away from the original built-up area exceeding $1 \mathrm{~km}$. In the PLAP figure, the high values were distributed in areas significantly closer to the original built-up than that of PLOP, which corresponded to the characteristics of adjacent or outlying urban growth.

In addition, MaxEnt also output the contributions of each variable to the result. Table 4 shows the relative contributions of environmental variables to PLOP and PLAP outputs. Percent contributions represented the normalized cumulative value of the variable gain in each iteration. Permutation importance (permutation importance) re-evaluated each environmental variable in sequence after random arrangement and displayed the AUC declines after training in the table and normalized them by percentage. 

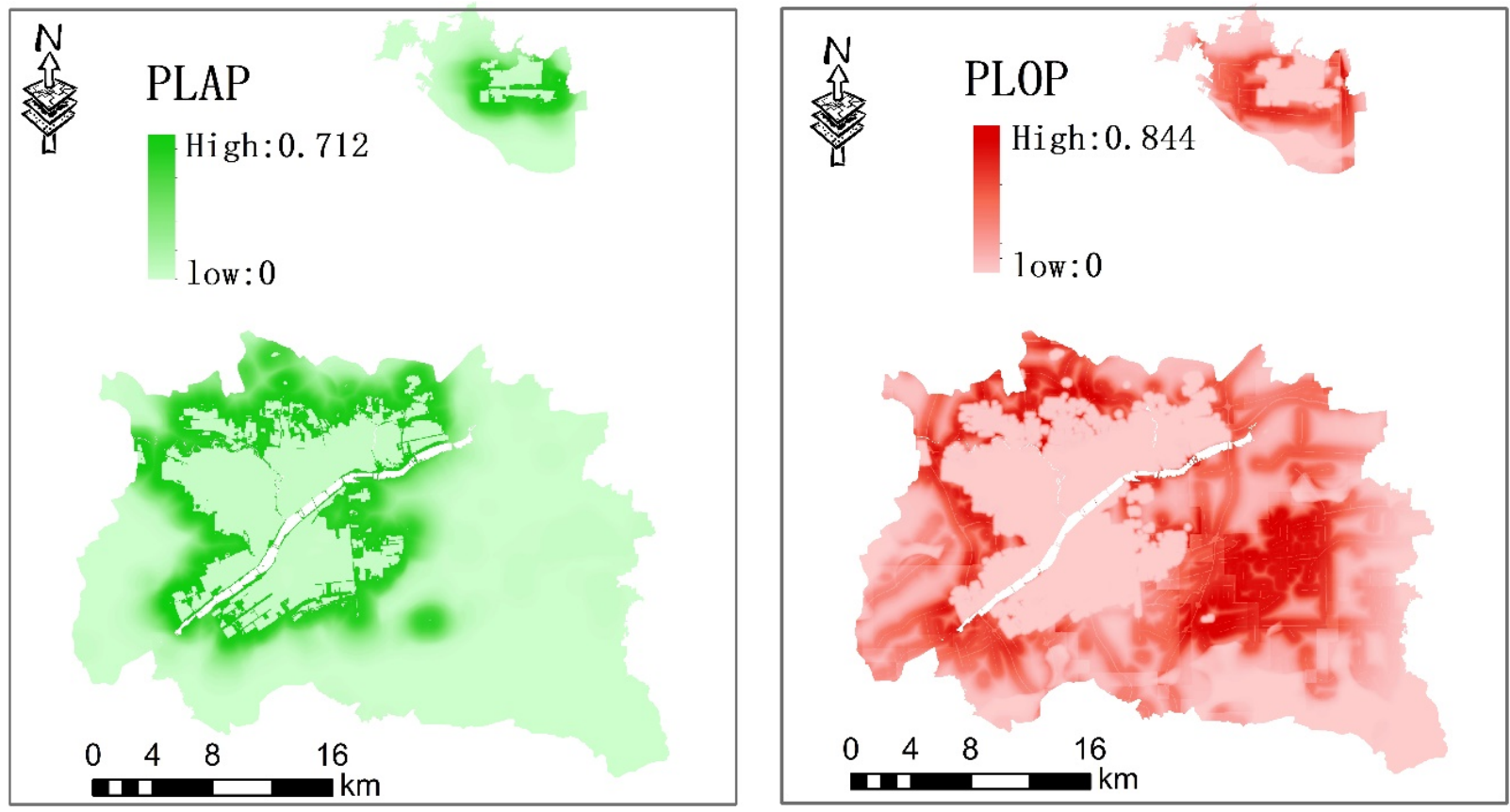

Figure 4. Spatial distributions of PLAP and PLOP.

Table 4. Variable Contributions for PLOP and PLAP.

\begin{tabular}{ccccc}
\hline \multirow{2}{*}{ Vibrable } & \multicolumn{2}{c}{ PLOP } & \multicolumn{2}{c}{ PLAP } \\
\cline { 2 - 5 } & $\begin{array}{c}\text { Percent } \\
\text { Contribution }\end{array}$ & $\begin{array}{c}\text { Permutation } \\
\text { Importance }\end{array}$ & $\begin{array}{c}\text { Percent } \\
\text { Contribution }\end{array}$ & $\begin{array}{c}\text { Permutation } \\
\text { Importance }\end{array}$ \\
\hline toBuilt & 38.4 & 28.6 & 53.6 & 67.6 \\
toRoad & 27.4 & 17.8 & 4.1 & 3.5 \\
toAirport & 14.6 & 6.4 & 0.6 & 3.1 \\
NFDensity & 3.9 & 16.7 & 34.7 & 13.7 \\
GDPDensity & 7.9 & 10.8 & 4.2 & 3.4 \\
toHRailStation & 4.1 & 12 & 0.5 & 4.9 \\
toCCenter & 3.2 & 1.1 & 2.1 & 0.6 \\
toPCenter & 0.5 & 6.5 & 0.2 & 3.2 \\
\hline
\end{tabular}

As the table shows, the variable contributions to PLOP and PLAP is different. The variables toBuilt, toRoad and toAirport had the most significant impact on the distribution of PLOP, while variables toBuilt, NFDensity and GDPDensity had the most significant impact on PLAP. It is worth noting that the variable toAirport had a significant contribution to the PLOP distribution but no obvious effect in the PLAP distribution. This suggests that in the future, driven by airport construction, more outlying growth may appear around the airport.

\subsection{Simulation Results and Accuracy Evaluation}

MaxEnt-CSCA generated more realistic local evolution rules for different types of urban growth patches to determine the temporal and spatial dynamics of urban expansion. GDAL and NumPy function libraries in the Python function library were used to read the starting year's image into a two-dimensional array, arrange the priority of the cells, judge the significance of the cell category and start the cell iteration process. After iteration, the urban growth patterns in the main urban area of Luoyang from 2009 to 2018 were simulated (see Figure 5). The overall accuracy was $87.65 \%$, the kappa coefficient was 0.78 , and Figure 5 shows, the simulation distribution was similar to the actual urban pattern. Both the statistics and the visual interpretation confirmed that the model achieved good results. Not only was there clear adjacent expansion in the relatively compact area, but 
outlying-type growth also appeared in areas away from the original urban patches, which was a "patchy-like" expansion rather than a complete "diffusion" state, that matched the actual situation. In about 10 years from 2009 to 2018, the actual areas expanded by adjacent and outlying cities were 24.64 and $13.10 \mathrm{~km}^{2}$, respectively, and the simulation results from MaxEnt-CSCA were 27.22 and $10.53 \mathrm{~km}^{2}$, respectively. This verified that the MaxEnt-CSCA model can accurately predict expansion trends of urban built-up in Luoyang.
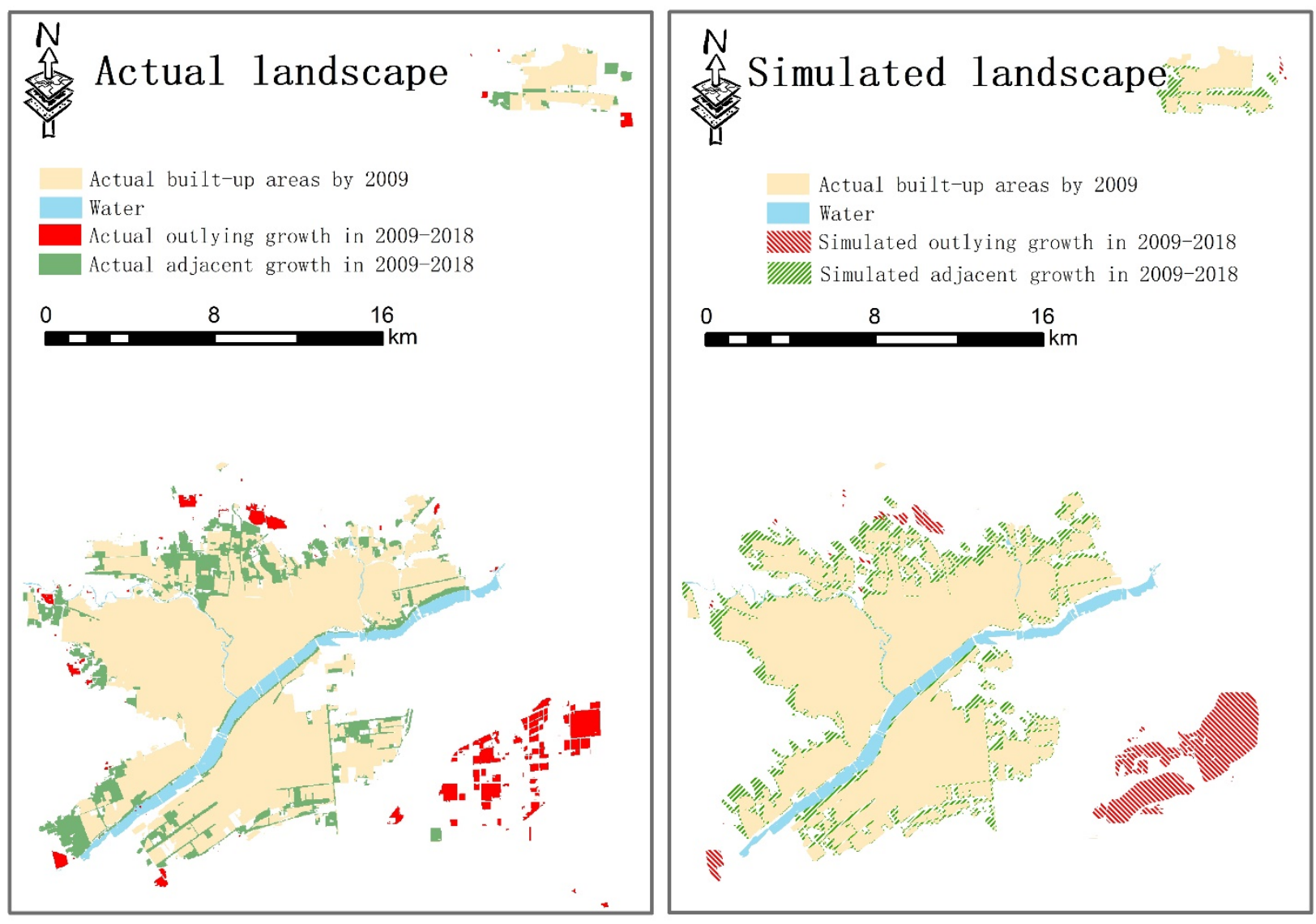

Figure 5. Actual and simulated urban landscapes of Luoyang's main urban areas by 2018.

\section{Discussion}

\subsection{Comparison with Other $C A$}

In simulating the historical evolution of Luoyang's urban expansion, the spatial output and several indicators of the MaxEnt-CSCA model achieved good results, but this did not indicate the advancement of the model. Only by comparing it with other models can the superiority of MaxEnt-CSCA model be confirmed. Therefore, this article continued to simulate the urban expansion of Luoyang City from 2009 to 2018 using two other CA models: MaxEnt-CA model, which considers the cellular conversion potential but does not consider the spatial zoning, and Base-CA model, which takes into account neither cellular conversion potential nor the spatial zoning. Maxent-CA still used the maximum entropy principle to obtain the probability of non-urban cells becoming urban cells and the selected environmental variables were the same to avoid subjectivity. By arranging the conversion probability from high to low and traversing each cell from non-urban to urban if the neighborhood condition was satisfied; Base-CA, on the other hand, only considering the neighborhood limit (there are enough construction land cells in the $3 \times 3$ neighborhood). The simulation results of the three models (Base-CA, Maxent-CA, Maxent-CSCA) were compared with the actual built-up areas in 2018. The result was shown in Figure 6. Noted that Jili District (2), which is far away from the main urban area, is not drawn in Figure 6 for visual aesthetics. In addition to spatial accuracy, Table 5 also compared the statistical 
accuracy, i.e., kappa coefficient and areas corrected simulated (the area of overlap between simulated landscape and actual landscape) of the three models.

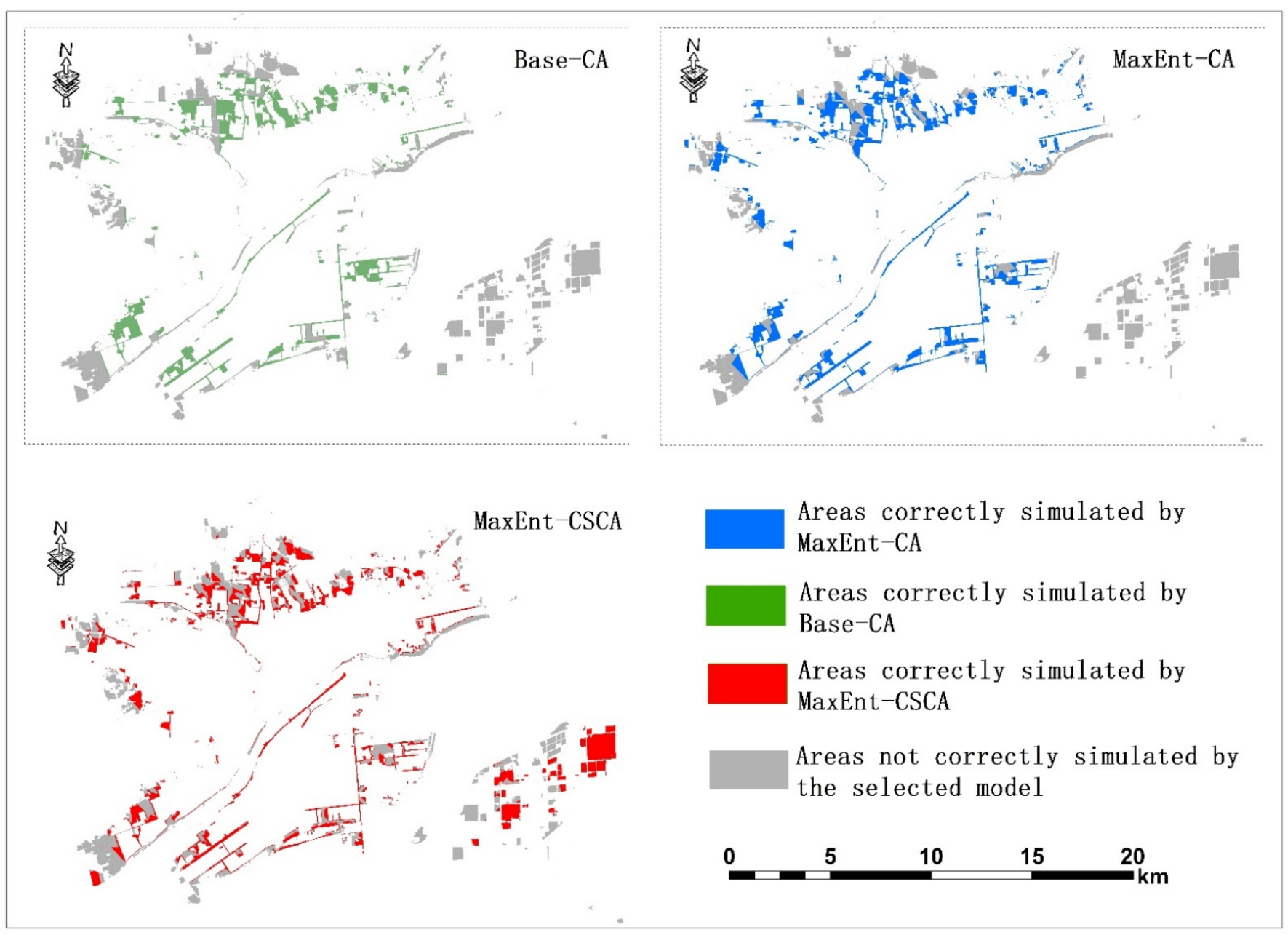

Figure 6. Comparison between the simulated landscapes by MaxEnt-CA and MaxEnt-CSCA.

Table 5. Comparison of kappa and areas corrected simulated among three models.

\begin{tabular}{cccc}
\hline \multirow{2}{*}{ Indicator } & \multicolumn{3}{c}{ Model Name } \\
\cline { 2 - 4 } & Base-CA & MaxEnt-CA & MaxEnt-CSCA \\
\hline Kappa & 0.71 & 0.74 & 0.78 \\
Areas corrected simulated $\left(\mathrm{km}^{2}\right)$ & 8.27 & 9.47 & 14.61 \\
\hline
\end{tabular}

The Maxent-CA results obtained a kappa coefficient of 0.74 , which was slightly less than MaxEnt-CSCA, and the corresponding value for Base-CA is only 0.71 , much lower than that for Maxent-CSCA. Landscape simulated by Base-CA and MaxEnt-CA represented a similar compact clustered phenomenon, where outlying growth was quite different from the actual distribution (for example, the majority of Luolong District's outlying growth). However, because Base-CA only considers the limitation of neighborhood, it is more inclined to look for the internal gap of original built-up areas or the sags of the urban exterior form for growth (i.e., infilling-type growth) than Maxent-CA, and it is easier to form a regular form. MaxEnt-CA, on the other hand, had some advantages in urban aggregation growth simulation, this aggregation growth includes not only infilling growth but also edge growth. However, neither Base-CA nor MaxEnt-CA is insufficient for diffusion simulation. In contrast, as shown in Figure 6, the clustering phenomenon in the output of MaxEnt-CSCA was not as pronounced as in the other two CA model, and simulation 
of outlying urban growth performed better. In the adjacent UGT simulation, correctly predicted areas (e.g., spatially coincident) by base-CA, MaxEnt-CA and MaxEnt-CSCA were $54.18 \%, 63.05 \%$ and $47.40 \%$ respectively, while in the outlying UGT simulation, they were $2.65 \%, 4.92 \%$ and $34.13 \%$ respectively. This showed that the Base-CA and Maxent-CA model, which considered neighborhood limitations, performed better than MaxEnt-CSCA when simulating adjacent UGTs, but predictive ability was inferior to MaxEnt-CSCA when simulating outlying UGTs. Combining the two categories together, MaxEnt-CSCA had a higher overall kappa coefficient than Base-CA and MaxEnt-CA. From this result, it can be seen that in the actual urban expansion simulations, the city's expansion mode and compactness played a significant role when choosing different models. In this case study, the main urban area of Luoyang had a clear diffusion pattern during the study period, so it was more appropriate to choose MaxEnt-CSCA.

\subsection{Potential Applications of MaxEnt-CSCA}

The MaxEnt-CSCA proposed in this paper can effectively simulate urban expansion diffusion and aggregation process simultaneously, while traditional CA models mostly only show considerable simulation capabilities for aggregation growth. As a matter of fact, diffusion growth is a common phenomenon in modern urban development and it is especially pronounced in the following four situations: (1) In the early stage of urban expansion, there are a large number of diffusion-type growth situations. The most typical theory is the urban growth phase theory, which states that urban development is a process from diffusion to aggregation. The spread of small spots eventually forms several larger interconnected spots. For example, literature [5] found that the growth of Huangpi District in Wuhan City, China from 1993 to 2023 matched this situation; (2) Natural conditions restrict the continuous growth of the city. For example, cities with complex mountainous terrain, such as the "mountain city" of Chongqing, Panzhihua, Zhangjiajie, and Lhasa in China, Rio de Janeiro in Brazil, and Zurich in Switzerland. Mountainous landscapes have blocked natural continuous growth and resulted in a relatively dispersed urban pattern; For example, the urban expansion of Lhasa during 1995-2015 was mainly represented as outlying-growth [7]. The other is cities with many lakes and rivers, which also blocks the continuous expansion of urban built-up areas. Taking Wuhan (who known as "city of lakes") in China as example, diffusion urban growth accounted for a large proportion from 1995-2005 [7]; (3) Diffusion-spreading growth cities are common in Europe and America. Raleigh in the United States had low-density sprawl along suburban roads [31], which is what we usually call the "reverse urbanization" process; (4) Cities with developed county economy. For example, in Suzhou and Wuxi in the southern Jiangsu Province of China, there are many outlying-expansion of industrial parks around these cities [12]. The author believes that diffuse growth cannot be ignored in these type of urban growth simulations, and MaxEnt-CSCA is expected to be well applied in these areas.

\subsection{Inspiration for Urban Planning}

The MaxEnt-CSCA model proposed in this paper has been verified with good performance for predicting urban expansion patterns. Based on the urban construction patterns in Luoyang in 2018, the MaxEnt-CSCA model was adopted to predict the distribution of urban built-up areas in 2035, which is the last year of the Chinese Plan, called Long-Range Objectives Through the Year 2035. The spatial distribution is shown in Figure 7. According to the current development trend, an outlying UGT area of $8.69 \mathrm{~km}^{2}$ and an adjacent UGT area of $66.11 \mathrm{~km}^{2}$ are expected to occur between 2018 and 2035. The diffusion growth will decrease significantly and the urban pattern will become more compact. Luolong District (5) will have a large area of adjacent urban expansion in the future, and the newly added urban patches will fill the gap of the internal voids left by the outlying-type urban growth from 2009 to 2018. Luoyang's urban form is expected to gradually mature in the next 15 years. With the development of the city, the urban expansion of Chanhe District (1), Jili District (2), Jianxi District (3), Laocheng District (4) and Xigong District (6) will mainly 
occur along the edges of existing urban patches, making the existing patches continue to grow larger. Outlying-type urban expansion will almost entirely occur in Laocheng District (4) and Luolong District (5), while the urban growth process will be different. The former outlying UGT will grow outside of the existing built-up and the latter will expand on the inner gap, which will first diffuse, and then merge together. It can be predicted that in the future, land resources available for urban development in Chanhe District (1), Jili District (2), Jianxi District (3), Laocheng District (4) and Xigong District (6) will decrease, which make the urban form very compact. Measures need to be taken to increase the degree of built-up intensive use to meet the needs of future social and economic development, and attention should be paid to the protection of agricultural land around these districts. Luolong District (5) is still rich in available land resources. As the city continues to expand in the future, Luolong District (5) will form a relatively independent built-up area and serve as Luoyang's second center, which will share in urban function and the pressure in center Luoyang that are excessively concentrated.

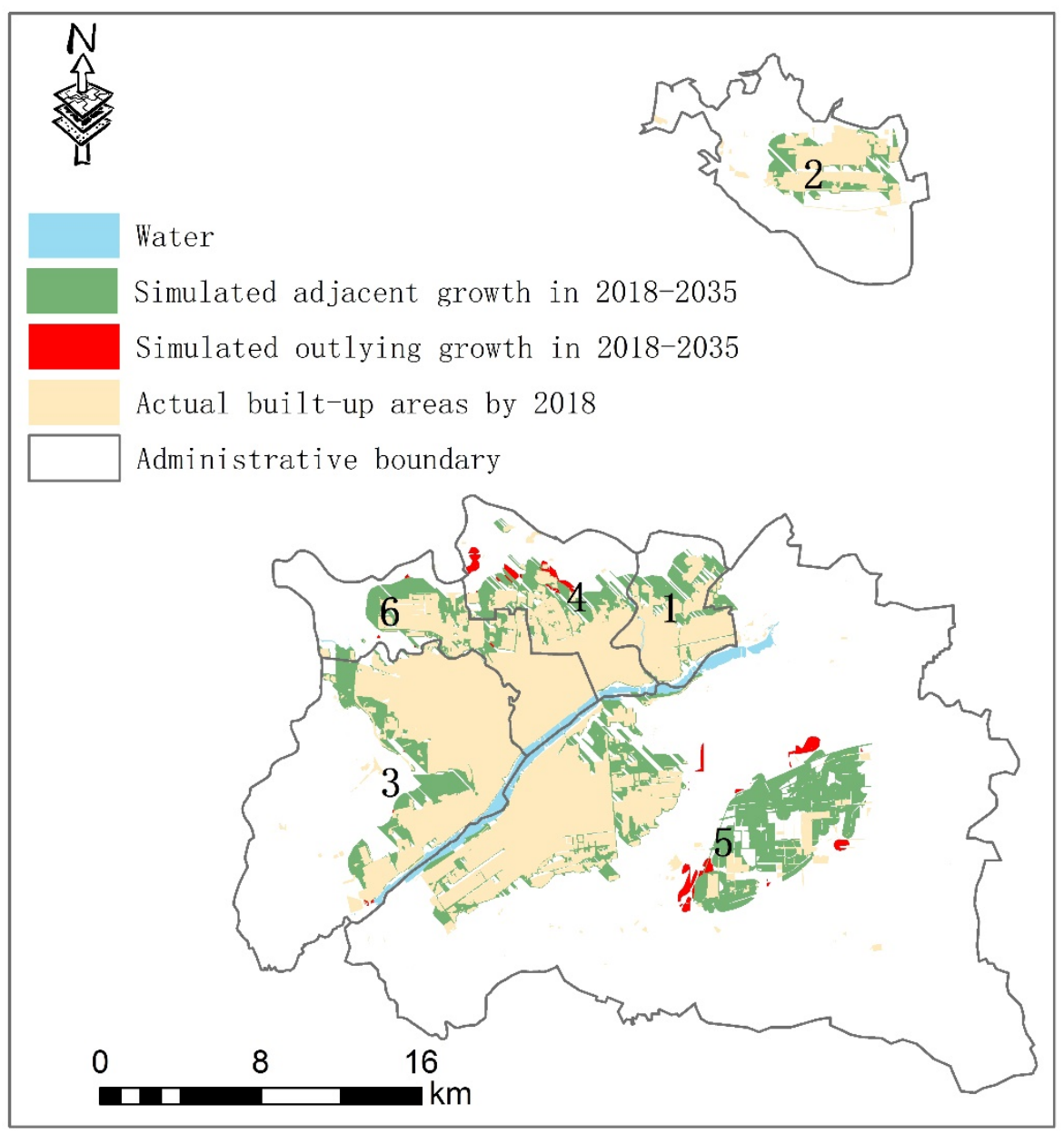

Figure 7. Predicted urban landscape in 2035 by MaxEnt-CSCA.

\section{Conclusions}

This paper proposed a category selection model, i.e., MaxEnt-CSCA model to simulate and predict urban expansion. This model made many improvements from the traditional cellular automata model, and expanded its hierarchical structure as well as the framework. It can simulate different urban growth categories using different local cell rules. The model of urban expansion and outlying-type urban expansion overcomes the shortcomings of the traditional CA model, which is unable to simulate diffuse outlying growth and adjacent urban sprawl simultaneously. The proposed model was verified using data from the main urban area of Luoyang between 2009 and 2018, where diffused urban expansion accounted 
for a large proportion. As we expected, the proposed MaxEnt-CSCA model effectively tracked the outlying built-up growth in Luoyang, which the traditional CA model was incapable of. Moreover, the MaxEnt-CSCA model was as good as the traditional CA model in terms of simulating adjacent growth. By predicting the spatial distribution of diffusion growth, it is helpful to predict the future urban growth center. Moreover, as the diffusional patch will continue to expand in the future, it will become an important carrier to undertake the spread of urban functions in the main urban area and provide choice for urban residents' real estate investment as well as the work opportunity. For future urban development, the gap between diffusional patches and original urban patches is the main place for future urban expansion and development, which is crucial for shaping and optimizing future urban form. Since urban form is associated with air quality [32], urban commuting [33], and spatial vitality [34] and so on, accurate simulation of the pattern and process of diffusional expansion patches can help decision makers to better formulate and optimize future urban form to achieve sustainable development. By further using MaxEnt-CSCA to predict and simulate the built-up landscape of Luoyang in 2035, it was found that the main urban area will continue to experience rapid urbanization, and future urban expansion will mainly occur in Luolong District and the counties surrounding it. In the near future, the urban pattern of Luolong District will grow from a relative diffusion state to a coalescing stage. Measures should be taken in these districts to make land use more efficient and intensive, and agricultural land surround the main urban areas should be protected. This paper verified the effectiveness of MaxEnt-CSCA through the case study of Luoyang, but as mentioned before, when performing spatial simulations of urban expansion for cities, the urban compactness as well as the city's development stage must be considered. Note that the proposed MaxEnt-CSCA model may not be superior in a city that is in the development stage of compact growth. Also, both the MaxEnt-CSCA and other traditional CA models have some fixed defects. For example, during simulation over a long time period, the environmental variables are assumed to be constant so that social and economic changes, such as newly built public facilities and an influx of floating population, will have no impact on the transformation of cells and thus reduce the simulated accuracy. Future research will focus on how to characterize the spatiotemporal changes of these socio-economic factors and couple their effects to MaxEnt-CSCA transformation rules.

Author Contributions: Conceptualization, R.W.; methodology, L.Z. and Y.L.; software, Y.L.; validation, W.H. and D.W.; formal analysis, L.Z.; resources, D.W.; data curation, W.H.; writing-original draft preparation, R.W. and W.H.; writing-review and editing, R.W. and L.Z.; supervision, Y.L. All authors have read and agreed to the published version of the manuscript.

Funding: This research was funded by National Natural Science Foundation of China (41801205).

Institutional Review Board Statement: Not applicable.

Informed Consent Statement: Not applicable.

Data Availability Statement: The data presented in this study are available on request from the corresponding author.

Conflicts of Interest: The authors declare no conflict of interest.

\section{References}

1. He, Q.; Tan, S.; Yin, C.; Zhou, M. Collaborative optimization of rural residential land consolidation and urban construction land expansion: A case study of Huangpi in Wuhan, China. Comput. Environ. Urban Syst. 2019, 74, 218-228. [CrossRef]

2. Li, H.; Liu, Y.; He, Q.; Peng, X.; Yin, C. Simulating urban cooperative expansion in a single-core metropolitan region based on improved ca model integrated information flow: Case study of Wuhan urban agglomeration in China. J. Urban. Plan. Dev. 2018, 144, 05018002. [CrossRef]

3. van Vliet, J.; White, R.; Dragicevic, S. Modeling urban growth using a variable grid cellular automaton. Comput. Environ. Urban Syst. 2009, 33, 35-43. [CrossRef]

4. Liu, X.; Ma, L.; Li, X.; Ai, B.; Li, S.; He, Z. Simulating urban growth by integrating landscape expansion index (LEI) and cellular automata. Int. J. Geogr. Inf. Sci. 2013, 28, 148-163. [CrossRef] 
5. Liu, Y.; He, Q.; Tan, R.; Liu, Y.; Yin, C. Modeling different urban growth patterns based on the evolution of urban form: A case study from Huangpi, Central China. Appl. Geogr. 2016, 66, 109-118. [CrossRef]

6. He, Q.; Liu, Y.; Zeng, C.; Chaohui, Y.; Tan, R. Simultaneously simulate vertical and horizontal expansions of a future urban landscape: A case study in Wuhan, Central China. Int. J. Geogr. Inf. Sci. 2017, 31, 1907-1928. [CrossRef]

7. He, Q.; Song, Y.; Liu, Y.; Yin, C. Diffusion or coalescence? Urban growth pattern and change in 363 Chinese cities from 1995 to 2015. Sustain. Cities Soc. 2017, 35, 729-739. [CrossRef]

8. Jiao, L.; Liu, J.; Xu, G.; Dong, T.; Gu, Y.; Zhang, B.; Liu, Y.; Liu, X. Proximity Expansion Index: An improved approach to characterize evolution process of urban expansion. Comput. Environ. Urban Syst. 2018, 70, 102-112. [CrossRef]

9. Dietzel, C.; Herold, M.; Hemphill, J.J.; Clarke, K.C. Spatio-temporal dynamics in California's Central Valley: Empirical links to urban theory. Int. J. Geogr. Inf. Sci. 2005, 19, 175-195. [CrossRef]

10. Liu, X.; Li, X.; Chen, Y.; Tan, Z.; Li, S.; Ai, B. A new landscape index for quantifying urban expansion using multi-temporal remotely sensed data. Landsc. Ecol. 2010, 25, 671-682. [CrossRef]

11. Clarke, K.C.; Hoppen, S.; Gaydos, L. A self-modifying cellular automaton model of historical urbanization in the San Francisco Bay area. Environ. Plan. B Plan. Des. 1997, 24, 247-261. [CrossRef]

12. He, Q.; Zhou, J.; Tan, S.; Song, Y.; Zhang, L.; Mou, Y.; Wu, J. What is the developmental level of outlying expansion patches? A study of 275 Chinese cities using geographical big data. Cities 2019, 105, 102395. [CrossRef]

13. Zheng, L.; Chen, K.; Tan, S.; Yin, C.; He, Q.; Wu, J. Landscape expansion-place quality nexus revisited: How fundamental and transitory growth impact in China? Land Use Policy 2021, 103, 105294. [CrossRef]

14. Jiao, L.; Mao, L.; Liu, Y. Multi-order Landscape Expansion Index: Characterizing urban expansion dynamics. Landsc. Urban Plan. 2015, 137, 30-39. [CrossRef]

15. Xia, C.; Zhang, A.; Yeh, A.G.-O. Shape-weighted landscape evolution index: An improved approach for simultaneously analyzing urban land expansion and redevelopment. J. Clean. Prod. 2019, 244, 118836. [CrossRef]

16. He, Q.; Zeng, C.; Xie, P.; Tan, S.; Wu, J. Comparison of urban growth patterns and changes between three urban agglomerations in China and three metropolises in the USA from 1995 to 2015. Sustain. Cities Soc. 2019, 50, 101649. [CrossRef]

17. Jaynes, E.T. Information Theory and Statistical Mechanics. Phys. Rev. 1957, 106, 620-630. [CrossRef]

18. Zhang, Y.; Liu, X.; Chen, G.; Hu, G. Simulation of urban expansion based on cellular automata and maximum entropy model. Sci. China Earth Sci. 2020, 63, 701-712. [CrossRef]

19. Holder, A.M.; Markarian, A.; Doyle, J.M.; Olson, J.R. Predicting geographic distributions of fishes in remote stream networks using maximum entropy modeling and landscape characterizations. Ecol. Model. 2020, 433, 109231. [CrossRef]

20. Yan, H.; Feng, L.; Zhao, Y.; Zhu, C.; Qu, Y.; Wang, H. Predicting the potential distribution of an invasive species, Erigeron canadensis L., in China with a maximum entropy model. Glob. Ecol. Conserv. 2019, 21, e00822. [CrossRef]

21. Zhang, K.; Zhang, Y.; Zhou, C.; Meng, J.; Sun, J.; Zhou, T.; Tao, J. Impact of climate factors on future distributions of Paeonia ostii across China estimated by MaxEnt. Ecol. Inform. 2019, 50, 62-67. [CrossRef]

22. Liu, Z.S.; Gao, H.; Teng, L.W.; Su, Y.; Wang, X.Q.; Kong, F.Y. Habitat suitability assessment of blue sheep in Helan Mountain based on MAXENT modeling. Acta Ecol. Sin. 2013, 33, 7243-7249. [CrossRef]

23. Liu, X.; Derudder, B.; Wang, M. Polycentric urban development in China: A multi-scale analysis. Environ. Plan. B Urban Anal. City Sci. 2017, 45, 953-972. [CrossRef]

24. Li, Y. Towards concentration and decentralization: The evolution of urban spatial structure of Chinese cities, 2001-2016. Comput. Environ. Urban Syst. 2019, 80, 101425. [CrossRef]

25. Tan, R.; Liu, Y.; Zhou, K.; Jiao, L.; Tang, W. A game-theory based agent-cellular model for use in urban growth simulation: A case study of the rapidly urbanizing Wuhan area of central China. Comput. Environ. Urban Syst. 2015, 49, 15-29. [CrossRef]

26. Firozjaei, M.K.; Sedighi, A.; Argany, M.; Jelokhani-Niaraki, M.; Arsanjani, J.J. A geographical direction-based approach for capturing the local variation of urban expansion in the application of CA-Markov model. Cities 2019, 93, 120-135. [CrossRef]

27. Li, X.; Zhou, W.; Ouyang, Z. Forty years of urban expansion in Beijing: What is the relative importance of physical, socioeconomic, and neighborhood factors? Appl. Geogr. 2013, 38, 1-10. [CrossRef]

28. Tan, R.; Liu, Y.; Liu, Y.; He, Q.; Ming, L.; Tang, S. Urban growth and its determinants across the Wuhan urban agglomeration, central China. Habitat Int. 2014, 44, 268-281. [CrossRef]

29. $\mathrm{Wu}, \mathrm{J} . ; \mathrm{Wu}, \mathrm{Y} . ; \mathrm{Yu}, \mathrm{W} . ; \mathrm{Lin}, \mathrm{J} . ; \mathrm{He}, \mathrm{Q}$. Residential landscapes in suburban China from the perspective of growth coalitions: Evidence from Beijing. J. Clean. Prod. 2019, 223, 620-630. [CrossRef]

30. Mcfadden, D. Modelling the choice of residential location. Transp. Res. Rec. 1978, 673, 72-77.

31. Dong, T.; Jiao, L.; Xu, G.; Yang, L.; Liu, J. Towards sustainability? Analyzing changing urban form patterns in the United States, Europe, and China. Sci. Total Environ. 2019, 671, 632-643. [CrossRef] [PubMed]

32. Mou, Y.; Song, Y.; Xu, Q.; He, Q.; Hu, A. Influence of Urban-Growth Pattern on Air Quality in China: A Study of 338 Cities. Int. J. Environ. Res. Public Health 2018, 15, 1805. [CrossRef] [PubMed]

33. Rao, Y.; Yang, J.; Dai, D.; Wu, K.; He, Q. Urban growth pattern and commuting efficiency: Empirical evidence from $100 \mathrm{Chinese}$ cities. J. Clean. Prod. 2021, 302, 126994. [CrossRef]

34. He, Q.; He, W.; Song, Y.; Wu, J.; Yin, C.; Mou, Y. The impact of urban growth patterns on urban vitality in newly built-up areas based on an association rules analysis using geographical 'big data'. Land Use Policy 2018, 78, 726-738. [CrossRef] 